\title{
AN ENERGETIC AFTERGLOW FROM A DISTANT STELLAR EXPLOSION
}

\author{
D. A. Frail, ${ }^{1}$ P. B. Cameron,${ }^{2}$ M. Kasliwal, ${ }^{2}$ E. Nakar,${ }^{3}$ P. A. Price, ${ }^{4}$ E. Berger,${ }^{5,6,7}$ A. Gal-Yam, ${ }^{2,7}$ S. R. Kulkarni, ${ }^{2}$ \\ D. B. Fox, ${ }^{8}$ A. M. Soderberg, ${ }^{2}$ B. P. Schmidt, ${ }^{9}$ E. Ofek, ${ }^{2}$ and S. B. Cenko ${ }^{10}$ \\ Received 2006 April 27; accepted 2006 June 15; published 2006 July 18
}

\begin{abstract}
We present the discovery of radio afterglow emission from the high-redshift $(z=6.295)$ burst GRB 050904 . The peak flux density for this burst is similar to typical low-redshift gamma-ray bursts (GRBs). We further show that beyond a redshift of order unity, the flux densities of radio afterglows are largely insensitive to redshift, consistent with predictions. By combining the existing X-ray, near-infrared, and radio measurements, we derive estimates for the kinetic energy and opening angle of the blast wave and for the density of the circumburst medium into which it expands. Both the kinetic and radiated energy indicate that GRB 050904 was an unusually energetic burst ( $\left.10^{52} \mathrm{ergs}\right)$. More importantly, we are able to make an in situ measurement of the density structure of the circumburst medium. We conclude that GRB 050904 exploded into a constant-density medium with $n_{0}=680 \mathrm{~cm}^{-3}$, which is 2 orders of magnitude above the nominal value for low-redshift GRBs. The next generation of centimeter (EVLA) and millimeter radio instruments (ALMA) will be able to routinely detect events such as GRB 050904 and use them to study magnetic fields and the atomic and molecular gas in the high-redshift universe.
\end{abstract}

Subject headings: cosmology: observations — gamma rays: bursts — radio continuum: general

\section{INTRODUCTION}

Understanding the reionization of the universe, when the first luminous sources were formed, is one of the latest frontiers of observational cosmology. Constraints have been obtained using diagnostics such as quasar studies of the Gunn-Peterson absorption trough, the luminosity evolution of Ly $\alpha$ galaxies, and the polarization isotropy of the cosmic microwave background. Taken together, these data portray a complicated picture in which reionization has taken place over a range of redshifts rather than at one specific epoch. The dominant source of reionization appears to be due to ultraviolet emission from young, massive stars (see review by Fan et al. 2006).

As the most luminous explosions in the universe, gammaray bursts (GRBs) are potential signposts of these early massive stars. The radio, infrared, and X-ray afterglow emission from GRBs are in principle observable out to $z \sim 30$ (Miralda-Escudé 1998; Lamb \& Reichart 2000; Ciardi \& Loeb 2000; Gou et al. 2004; Ioka \& Mészáros 2005). It is estimated that $10 \%$ of GRBs detected by the Swift satellite are at $z>5$ (Natarajan et al. 2005; Bromm \& Loeb 2006). The most distant GRB to date is GRB 050904 at $z=6.295$. It was detected by the Swift Burst Alert Telescope and localized to an accuracy of a few

\footnotetext{
${ }^{1}$ National Radio Astronomy Observatory, P.O. Box O, Socorro, NM 87801.

${ }^{2}$ Division of Physics, Mathematics, and Astronomy, MS 105-24, California Institute of Technology, Pasadena, CA 91125.

${ }^{3}$ Theoretical Astrophysics, California Institute of Technology, MS 130-33, Pasadena, CA 91125.

${ }^{4}$ Institute for Astronomy, University of Hawaii, 2680 Woodlawn Drive, Honolulu, HI 96822.

${ }^{5}$ Observatories of the Carnegie Institution of Washington, 813 Santa Barbara Street, Pasadena, CA 91101.

${ }^{6}$ Princeton University Observatory, Peyton Hall, Ivy Lane, Princeton, NJ 08544.

${ }^{7}$ Hubble Fellow.

${ }^{8}$ Department of Astronomy and Astrophysics, Pennsylvania State University, 525 Davey Laboratory, University Park, PA 16802.

${ }^{9}$ Research School of Astronomy and Astrophysics, Australian National University, Mount Stromlo Observatory, via Cotter Road, Weston Creek, ACT 2611, Australia.

${ }^{10}$ Space Radiation Laboratory, MS 220-47, California Institute of Technology, Pasadena, CA 91125.
}

arcseconds by the Swift X-Ray Telescope (Gehrels et al. 2004). Follow-up optical and near-infrared (NIR) observations were begun shortly thereafter. Details on the discovery and properties of the X-ray and NIR afterglow of this burst can be found in several papers (Cusumano et al. 2006; Watson et al. 2006; Haislip et al. 2006; Boër et al. 2006; Tagliaferri et al. 2005). An optical/NIR spectrum taken by the Subaru Telescope 3.4 days after the burst showed multiple heavy metal absorption lines and a Gunn-Peterson trough with a damping wing redward of the Ly $\alpha$ cutoff that was used to derive the neutral fraction $x_{\mathrm{H}}<0.6$ (Totani et al. 2006; Kawai et al. 2006). Recent observations with the Hubble Space Telescope and Spitzer revealed a faint host galaxy underlying the GRB position, with a luminosity of about $L^{*}$ and a star formation rate of $\sim 15 M_{\odot}$ $\mathrm{yr}^{-1}$ (Berger et al. 2006). With a Swift GRB detection rate of $100 \mathrm{yr}^{-1}$, GRBs could one day replace quasars as the preferred probe of the high-redshift universe.

In this paper we report on the detection of the radio afterglow from GRB 050904 ( $(2)$, which makes it possible to derive physical properties of the explosion and the circumburst medium $(\S 3)$. Our results are compared with predictions for the properties of the explosion and how the progenitors of highredshift GRBs are expected to have shaped their surrounding environments (\$4).

\section{OBSERVATIONS AND RESULTS}

Radio observations were undertaken with the Very Large Array $^{11}$ (VLA) at a frequency of $8.46 \mathrm{GHz}$ (see Table 1). To maximize sensitivity, the full VLA continuum bandwidth (100 $\mathrm{MHz}$ ) was recorded in two $50 \mathrm{MHz}$ bands. Data reduction was carried out following standard practice in the AIPS software package. Some additional phase and amplitude self-calibration was necessary to remove the contaminating effects of a bright (10 mJy) radio source $4^{\prime}$ northeast from the GRB.

Radio emission from GRB 050904 was not detected during

\footnotetext{
${ }^{11}$ The Very Large Array is operated by the National Radio Astronomy Observatory, a facility of the National Science Foundation operated under cooperative agreement by Associated Universities, Inc.
} 
TABLE 1

Radio ObSERVATIONS

\begin{tabular}{crr}
\hline \hline $\begin{array}{c}\text { Observation Date } \\
(\mathrm{UT})\end{array}$ & $\begin{array}{c}\Delta t \\
(\text { days })\end{array}$ & \multicolumn{1}{c}{$\begin{array}{c}F_{\nu}^{\mathrm{a}} \\
(\mu \mathrm{Jy})\end{array}$} \\
\hline 2005 Sep $4.58 \ldots \ldots$. & 0.50 & $89 \pm 58$ \\
2005 Sep $5.48 \ldots \ldots$. & 1.40 & $41 \pm 25$ \\
2005 Sep $9.48 \ldots \ldots$. & 5.40 & $-3 \pm 25$ \\
2005 Sep $10.31 \ldots \ldots$. & 6.23 & $27 \pm 24$ \\
2005 Sep $24.20 \ldots \ldots$ & 20.12 & $89 \pm 37$ \\
2005 Oct $3.36 \ldots \ldots$. & 29.28 & $40 \pm 30$ \\
2005 Oct $7.30 \ldots \ldots$. & 33.22 & $-10 \pm 35$ \\
2005 Oct $8.26 \ldots \ldots$. & 34.18 & $64 \pm 23$ \\
2005 Oct $9.34 \ldots \ldots$. & 35.26 & $116 \pm 18$ \\
2005 Oct $11.43 \ldots \ldots$. & 37.35 & $67 \pm 17$ \\
2005 Oct $18.37 \ldots \ldots$. & 44.29 & $13 \pm 27$ \\
\hline
\end{tabular}

${ }^{a}$ All errors are given as $1 \sigma(\mathrm{rms})$.

the first week, ruling out a bright short-lived component similar to GRB 990123 (Kulkarni et al. 1999). By averaging the data from this period, we obtain a peak flux at the position of the NIR afterglow of $33 \pm 14 \mu \mathrm{Jy}$. A clear detection was made during three epochs 34-37 days after the burst (see Table 1). Averaging the data taken in October, we obtain a mean flux density of $76 \pm 14 \mu \mathrm{Jy}$. The spectral radio luminosity, expressed as $L_{\nu}=4 \pi F_{\nu} d_{L}^{2}(1+z)^{\alpha-\beta-1}=2.5 \times 10^{31} \mathrm{ergs} \mathrm{s}^{-1}$ $\mathrm{Hz}^{-1}$ (where $F_{\nu} \propto t^{\alpha} \nu^{\beta}$ and $\alpha \sim 0$ and $\beta=1 / 3$ have been assumed, corresponding to an optically thin, flat, post-jet-break light curve), is normal for GRBs (Soderberg et al. 2004) and is 2 orders of magnitude below the highest redshift radio-loud quasars (Carilli et al. 2004).

We show these detections in Figure 1, together with a complete sample of $8.5 \mathrm{GHz}$ flux density measurements for GRBs with known redshifts. Most GRB radio afterglows with known redshifts have peak flux densities in the range of 100-1000 $\mu \mathrm{Jy}$. Likewise, in the source rest frame the peak flux density for the majority of afterglows is reached around 5 days postburst (Frail 2005). Neither the time to peak nor the flux density of GRB 050904 is unusual compared to this known sample of GRBs at lower redshifts. More interestingly, the average centimeter flux density in Figure 1 shows only a weak dependence on redshift.

This effect was predicted by Ciardi \& Loeb (2000) and is shown here for the first time. It is reminiscent of the "negative $k$-correction" for submillimeter observations of ultraluminous infrared galaxies (Blain et al. 2002). The afterglow flux density remains high because of the dual effects of spectral and temporal redshift, offsetting the dimming due to the increase in distance (Lamb \& Reichart 2000). We illustrate in Figure 1 how slowly the centimeter flux density decreases for a canonical GRB afterglow beyond $z \sim 1$ due to this effect (longdashed lines), compared to one whose luminosity is assumed constant (short-dashed lines). Observational bias does not explain the flattening in Figure 1 because the detection rate of radio afterglows is largely insensitive to redshift. Of the 60 GRBs with known redshift that have been observed in the radio, there are 42 with detected afterglows. Their detection rates above and below $z=1$ are identical.

\section{AFTERGLOW MODELING}

We now proceed to combine these radio data $(\S 2)$ with the extensive X-ray and optical/NIR data for GRB 050904 in order to constrain the physical parameters of the outflow and the circumburst medium. We interpret these multiwavelength data within the framework of the relativistic blast wave model (see

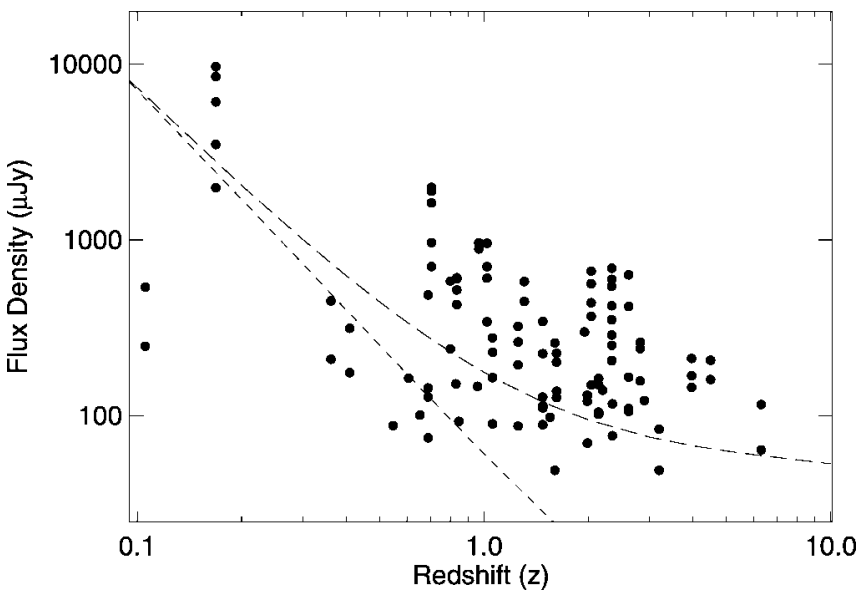

FIG. 1.-Radio flux density vs. redshift from a complete sample of 60 bursts observed at radio wavelengths from 1997 to 2006. The radio measurements were made at $8.5 \mathrm{GHz}$ and are taken from Frail et al. (2003) and the public radio afterglow database (http://www.aoc.nrao.edu/ dfrail/grb_public.shtml). For clarity only the 42 GRBs with known redshifts and radio afterglow detections are plotted with measurements taken less than 5 days after the burst in its rest frame. The long-dashed line is the flux density for a canonical afterglow model at $\Delta t=5$ days (in the observer frame). It assumes $E_{k, \text { iso, } 52}=10^{53} \mathrm{ergs}, \theta_{j}=0.1 \mathrm{rad}, n_{0}=10 \mathrm{~cm}^{-3}, p=2.2, \epsilon_{e}=0.1$, and $\epsilon_{B}=1 \%$ (see text for more details). The short-dashed line shows the expected decrease if the flux scales simply as the inverse square of the luminosity distance.

Mészáros 2002 for a review). In brief, the evolution of the broadband spectrum and hence the light curves are governed by the isotropic kinetic energy of the shock $E_{k, \text { iso, } 52}$, the opening angle of the jet $\theta_{j}$, the density of the circumburst medium $n_{0}$, the electron energy index $p$, and the fraction of the shock energy density in relativistic electrons $\epsilon_{e}$ and magnetic fields $\epsilon_{B}$. This particular fitting approach is described in more detail in Yost et al. (2003).

To limit the number of free parameters in the fit, we chose to model only the evolution of the forward shock after energy injection has ceased. The early X-ray light curve shows complex flaring activity, common in Swift bursts but lasting an unusually long time ( $\Delta t<0.7$ days), corresponding to a centralengine lifetime of more than $2 \mathrm{hr}$ in the source rest frame (Cusumano et al. 2006). Ongoing energy injection is also suspected based on the flares and the flattening of the optical/NIR light curves on similar timescales (Boër et al. 2006; Haislip et al. 2006). If these early data are excluded the NIR data show a smooth power-law evolution with a clear break at $t_{j}=$ $2.6 \pm 1.0$ days and decay indices before and after the break of $\alpha_{1}=-0.72_{-0.20}^{+0.15}$ and $\alpha_{2}=-2.4 \pm 0.4$ (Tagliaferri et al. 2005). If interpreted as a jet break, the sharpness of the transition makes it unlikely that this burst occurred in a windblown environment (Kumar \& Panaitescu 2000) and motivates our modeling choice of a constant-density circumburst medium (see also Gendre et al. 2006).

Our best-fit forward-shock model is shown in Figure 2. Our model makes the unusual prediction that the synchrotron cooling frequency lies below both the X-ray and optical bands before the first $J$-band detection of the afterglow at $\Delta t=$ $3.07 \mathrm{hr}$ (Haislip et al. 2006). This implies a steep spectral slope of $\beta=-p / 2=-1.07$ (Sari et al. 1999). This is likely the case at $\Delta t=3$ days, where we derive an NIR/X-ray slope of $\beta_{\text {ox }}=-1.1 \pm 0.05$. This value also agrees with Tagliaferri et al. (2005), who derive a best-fit optical/NIR spectral index of $\beta_{o}=-1.25 \pm 0.25$ over the interval from 0.4 to 7 days post- 

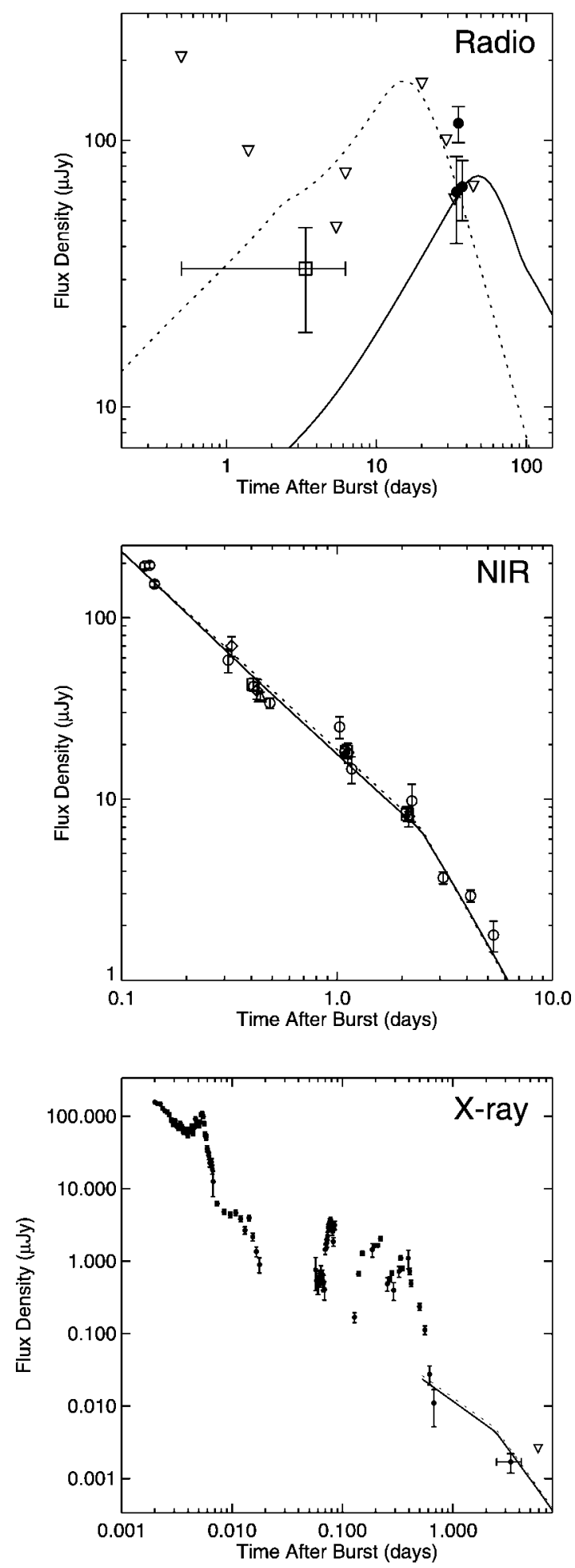

FIG. 2.-Broadband afterglow fits for the radio, NIR, and X-ray data of GRB 050904. The radio data are taken from $\S 2$. Filled circles indicate detections, and upper limits are shown as inverted triangles (plotted as flux + $2 \sigma$ ). The open square is the peak flux obtained by averaging the first week of data. The NIR data are taken from Tagliaferri et al. (2005) and Haislip et al. (2006), with a small correction made for Galactic dust extinction (Schlegel et al. 1998) before converting to flux density (Bessell et al. 1998). JHK and $K^{\prime}$ are shown for display purposes on the same plot after scaling by the bestfit spectral index. We have converted the Swift X-ray data $(0.2-10 \mathrm{keV})$ to flux density using the average photon index $\Gamma=-1.84$ and a frequency of $2.8 \times 10^{17} \mathrm{~Hz}$ (see Cusumano et al. 2006). The solid line is our best-fit forwardshock model for a constant-density circumburst medium with $E_{k, \text { iso, } 52}=$ $8.8 \times 10^{53} \mathrm{ergs}, \theta_{j}=0.14 \mathrm{rad}, n_{0}=680 \mathrm{~cm}^{-3}, p=2.14, \epsilon_{e}=2.0 \%$, and $\epsilon_{B}=1.5 \%$ (see text for more details). The thin dashed line shows the effect of model fitting when the value of $n_{0}$ is fixed at 100 times lower than the bestfit value. burst. A steep spectral slope of $\beta_{x} \sim-0.85$ is seen even when the $\mathrm{X}$-ray light curve is highly time variable in the range 0.05 days $\leq \Delta t \leq 0.7$ days. Thus we find that the available data support a small value for the cooling frequency.

Our best-fit model favors a circumburst density $n_{0}=680$ $\mathrm{cm}^{-3}$. To test the robustness of this result we ran several model fits, fixing $n_{0}$ over a range of values between 0.7 and $7 \times$ $10^{4} \mathrm{~cm}^{-3}$. As expected, the X-ray and NIR data are relatively insensitive to $n_{0}$, so the radio afterglow provides the tightest constraints. Large densities $n_{0}>10^{4} \mathrm{~cm}^{-3}$ are ruled out since the solutions are bounded by the condition that the magnetic and electron densities do not exceed equipartition. Low densities $n_{0}<10 \mathrm{~cm}^{-3}$ are also not favored since they predict bright early radio emission in the first week well in excess of that which is observed ( $(2)$. Over the allowable density range, $E_{k \text {, iso, } 52}$ varies from 40 to 530 , and therefore it is no better constrained by the afterglow data than the isotropic radiated energy $E_{\gamma, \text { iso, 52 }}$ is by the prompt emission (Cusumano et al. 2006).

Another possibility is that radio afterglow flux density measurements are biased by the turbulent ionized interstellar medium of our Galaxy, which induces non-Gaussian intensity fluctuations for compact radio sources. Indeed, it has been shown by Cordes \& Lazio (1991) that repeated short observations similar to those in Table 1 increase the odds of detecting a weak radio signal close to the detection threshold. We replaced the detections with upper limits and reran the fit. High-density solutions are still preferred $\left(n_{0} \simeq 10^{4} \mathrm{~cm}^{-3}\right)$ because both the X-ray and optical afterglows of GRB 050904 were bright (Cusumano et al. 2006; Haislip et al. 2006). Thus, in order to suppress the radio emission at $8.5 \mathrm{GHz}$ (or $62 \mathrm{GHz}$ in the rest frame), a large value of the synchrotron self-absorption is required and hence a large circumburst density.

\section{DISCUSSION}

With the detection of the radio afterglow from GRB 050904 , we have a complete multiwavelength data set for this highredshift burst. By comparing the physical properties of the burst and its circumburst environment to those GRBs found at lower redshifts, we hope to gain some insight into the birth and death of the earliest generations of stars in the universe.

There is good support for interpreting GRB 050904 as an energetic event. From our afterglow modeling ( $\S 3$ ) of the isotropic kinetic energy of the shock, we have $E_{k \text {, iso, 52 }}=88$ (normalized in units of $10^{52} \mathrm{ergs}$ ). Estimates obtained for the isotropic radiated energy (Cusumano et al. 2006) are in the range $66 \leq E_{\gamma \text {, iso, 52 }} \leq 320$. The quoted range in $E_{\gamma \text {, iso, } 52}$ reflects the uncertainty in the location of the peak of the gamma-ray spectrum. These constraints can be refined owing to our fitting the jet opening angle $\theta_{j}=0.14 \mathrm{rad}$ (see also Tagliaferri et al. 2005). The geometrically corrected energies are $E_{k, 52} \simeq$ $E_{k \text {,iso, } 52} \times \theta_{j}^{2} / 2=0.9$, and $0.7 \leq E_{\gamma, 52} \leq 3$. Both the radiated and kinetic energy of this event lie at or beyond the values derived for a large sample of lower redshift afterglows (Panaitescu \& Kumar 2001, 2002; Yost et al. 2003; Friedman \& Bloom 2005). An independent check on this result uses the $X$ ray light curve to compute the geometrically corrected X-ray luminosity at some fiducial time (usually taken at $\Delta t=10 \mathrm{hr}$ ). We derive $L_{\mathrm{X}}=2.6 \times 10^{45} \mathrm{ergs} \mathrm{s}^{-1}$, again larger than any previous GRB afterglow (Berger et al. 2003).

Perhaps the most interesting aspect of these data is that they allow us to make an in situ measurement of the density structure for a massive star in the early universe. From our afterglow 
modeling we conclude that GRB 050904 exploded into a constant-density medium with $n_{0}=680 \mathrm{~cm}^{-3}$, which is 2 orders of magnitude above the nominal value for lower redshift GRBs (Friedman \& Bloom 2005; Soderberg et al. 2006). Line-ofsight measures (Watson et al. 2006; Haislip et al. 2006; Totani et al. 2006) also indicate that a substantial column of gas exists toward GRB 050904. The fine-structure silicon lines Si II* are the most important since their column density ratios yield estimates of the electron density $n_{e} \sim 10^{2.3 \pm 0.7} \mathrm{~cm}^{-3}$ (Kawai et al. 2006). The close agreement of this line-of-sight estimate with our in situ value argues for a common physical origin for the gas in the immediate vicinity of the GRB.

There are testable predictions for star formation and collapse in the early universe (Bromm \& Loeb 2006 and reference therein). The first stars are expected to be very massive and could produce energetic GRB explosions like GRB 050904. Likewise, the high density we derived for GRB 050904 may be part of the general increase expected for the ambient density of the interstellar medium (Ciardi \& Loeb 2000), but more likely just indicates that this GRB exploded in a dense molecular cloud. A lower density would be expected if the progenitor of GRB 050904 was a low-metallicity Wolf-Rayet star (Vink $\&$ de Koter 2005). However, it remains a problem, at both low and high redshifts, why the radial density signature from the collapsar wind is rarely detected (Chevalier et al. 2004). A larger sample is needed before drawing too much from these predictions. Deep radio observations will continue to play a role since GRBs can be seen out to the highest redshifts and are sensitive probes of the total calorimetry of the explosion and the density structure of the circumburst medium. Looking further ahead to the next generation of centimeter (EVLA) and millimeter radio instruments (ALMA), events such as GRB 050904 will be bright enough to sample primordial magnetic fields (Gaensler et al. 2004) and probe the cold atomic and molecular gas from the time of the first light (Inoue et al. 2005).

D. A. F. wishes to thank B. Clark for his generous allocation of VLA time during the dynamic scheduling tests.

\section{REFERENCES}

Berger, E., Kulkarni, S. R., \& Frail, D. A. 2003, ApJ, 590, 379

Berger, E., et al. 2006, ApJ, submitted (astro-ph/0603689)

Bessell, M. S., Castelli, F., \& Plez, B. 1998, A\&A, 333, 231

Blain, A. W., Smail, I., Ivison, R. J., Kneib, J.-P., \& Frayer, D. T. 2002, Phys. Rep., 369, 111

Boër, M., Atteia, J. L., Damerdji, Y., Gendre, B., Klotz, A., \& Stratta, G. 2006, ApJ, 638, L71

Bromm, V., \& Loeb, A. 2006, ApJ, 642, 382

Carilli, C. L., et al. 2004, AJ, 128, 997

Chevalier, R. A., Li, Z., \& Fransson, C. 2004, ApJ, 606, 369

Ciardi, B., \& Loeb, A. 2000, ApJ, 540, 687

Cordes, J. M., \& Lazio, T. J. 1991, ApJ, 376, 123

Cusumano, G., et al. 2006, Nature, 440, 164

Fan, X., Carilli, C. L., \& Keating, B. 2006, ARA\&A, 44, 415

Frail, D. A. 2005, in IAU Colloq. 192, Cosmic Explosions: On the 10th Anniversary of SN 1993J, ed. J.-M. Marcaide \& K. W. Weiler (Berlin: Springer), 451

Frail, D. A., Kulkarni, S. R., Berger, E., \& Wieringa, M. H. 2003, AJ, 125, 2299

Friedman, A. S., \& Bloom, J. S. 2005, ApJ, 627,

Gaensler, B. M., Beck, R., \& Feretti, L. 2004, NewA Rev., 48, 1003

Gehrels, N., et al. 2004, ApJ, 611, 1005

Gendre, B., et al. 2006, A\&A, submitted (astro-ph/0603431)

Gou, L. J., Mészáros, P., Abel, T., \& Zhang, B. 2004, ApJ, 604, 508
Haislip, J. B., et al. 2006, Nature, 440, 181

Inoue, S., Omukai, K., \& Ciardi, B. 2005, MNRAS, submitted (astro-ph/ 05022180)

Ioka, K., \& Mészáros, P. 2005, ApJ, 619, 684

Kawai, N., et al. 2006, Nature, 440, 184

Kulkarni, S. R., et al. 1999, ApJ, 522, L97

Kumar, P., \& Panaitescu, A. 2000, ApJ, 541, L9

Lamb, D. Q., \& Reichart, D. E. 2000, ApJ, 536, 1

Mészáros, P. 2002, ARA\&A, 40, 137

Miralda-Escudé, J. 1998, ApJ, 501, 15

Natarajan, P., Albanna, B., Hjorth, J., Ramirez-Ruiz, E., Tanvir, N., \& Wijers, R. 2005, MNRAS, 364, L8

Panaitescu, A., \& Kumar, P. 2001, ApJ, 554, 667 2002, ApJ, 571, 779

Sari, R., Piran, T., \& Halpern, J. P. 1999, ApJ, 519, L17

Schlegel, D. J., Finkbeiner, D. P., \& Davis, M. 1998, ApJ, 500, 525

Soderberg, A. M., Nakar, E., Berger, E., \& Kulkarni, S. R. 2006, ApJ, 638, 930

Soderberg, A. M., et al. 2004, ApJ, 606, 994

Tagliaferri, G., et al. 2005, A\&A, 443, L1

Totani, T., et al. 2006, PASJ, 58, 485

Vink, J. S., \& de Koter, A. 2005, A\&A, 442, 587

Watson, D., et al. 2006, ApJ, 637, L69

Yost, S. A., Harrison, F. A., Sari, R., \& Frail, D. A. 2003, ApJ, 597, 459 